\title{
Transfection microarray of nonadherent cells on an oleyl poly(ethylene glycol) ether-modified glass slide
}

\author{
Koichi Kato ${ }^{1}$, Kohei Umezawa ${ }^{2}$, Masato Miyake ${ }^{1}$ Jun Miyake , and Teruyuki Nagamune ${ }^{2}$
}

BioTechniques 37:444-452 (September 2004)

\begin{abstract}
Cell-based microarrays are emerging as a tool for analyzing the functions of genes in cells. However, partly due to the difficulty of cell immobilization, the application of this method has been limited to adherent cells. We previously reported a method that rapidly and strongly attached living nonadherent cells to glass slides modified with a cell membrane anchoring reagent, designated a biocompatible anchor for membrane (BAM). Here we demonstrate that plasmid DNA deposited in a defined area on BAM-modified glass slides was transfected into nonadherent K562 cells immobilized on the DNA-deposited and BAM-modified slides. This method allowed the transfection of K562 cells not only with plasmid cDNA expression vectors but also with small interfering RNA (siRNA) at a defined location on the BAM-modified slides. We expect this methodology to greatly expand the scope of current cell microarray technology.
\end{abstract}

\section{INTRODUCTION}

A large amount of information on genomic sequences is available, but daunting tasks remain in the study of the functions and interactions of proteins expressed in cells. To efficiently analyze the functions of genes in cells, cell-based microarrays have recently been developed and have now emerged as a valuable tool. These microarrays are remarkable for high performance of thousands of experiments on a highly parallelized and miniaturized scale (1-7). Ziauddin and Sabatini (7) demonstrated simultaneous analyses of the functions of hundreds of genes in human embryonic kidney (HEK)293T cells on a glass slide. Microarrays of living mammalian cell clusters that express a defined cDNA at each location have been prepared by microprinting sets of cDNAs cloned in expression vectors. This is a very attractive method for studying the functions of gene products on a genomewide scale. In these cell microarray systems, however, the adhesive characteristics of adherent cells have been utilized to attach the cells to the glass slides. Therefore, a major limitation of the cell microarray methods is that they cannot be applied to nonadherent cells, such as blood cells (especially immunocytes), some stem cells, and lines of cancer cells, all of which are very important in the various biological and medical fields.

Although cell-based analysis, including the use of nonadherent cells, has generally been conducted in culture dishes or in 96- and 384-well microplates, the microarray platform has distinct advantages over the wellbased one. For example, microarrays would be significantly more effective for high-throughput analysis because the sample density is hundreds of times higher than that of a microplate. This feature is brought about by the small culture scale. Furthermore, miniaturization allows for cost-effective assays and requires minimal amounts of materials (e.g., nucleic acids, transfection reagents, and cells) $(1,3,4,6)$. Such benefits would be very attractive for assays of nonadherent cells.

In our previous study, we demon- strated that nonadherent cells could be immobilized on biocompatible anchor for membrane (BAM)-modified glass supports and proliferate normally. This result indicated that BAM could be used to create microarrays of nonadherent cells (8). In this report, by using this technique for the culture of immobilized nonadherent cells, we aimed to develop a gene transfection microarray for nonadherent cells on DNA preprinted and BAM-modified glass slides.

\section{MATERIALS AND METHODS}

\section{Materials}

Bovine serum albumin (BSA; low endotoxin) and RPMI 1640 culture medium were purchased from Sigma Chemical (St. Louis, MO, USA); Lipofectamine ${ }^{\mathrm{TM}}$ 2000 (abbreviated here as LF2000) and phosphate-buffered saline (PBS) were from Invitrogen (Carlsbad, CA, USA); fetal bovine serum (FBS) was from Dainippon Pharmaceuticals Co. Ltd. (Osaka, Japan); glass slides were from Matsunami Glass Ind. Ltd. (Osaka, Japan).

${ }^{1}$ National Institute of Advanced Industrial Science and Technology, Amagasaki and ${ }^{2}$ The University of Tokyo, Tokyo, Japan 


\section{BAM Reagents}

Oleyl-O-poly(ethylene glycol)-succinyl-N-hydroxy-succinimidyl esters [the average number of ethylene oxide unit repeats was 180; BAM180] were gifts from the NOF Corporation (Tokyo, Japan). A commercially available equivalent of BAM180 is sold by NOF under the product name SUNBRIGHT OE-080CS.

\section{Cell Lines}

The K562 human erythroleukemic cell line was obtained from the Institute of Development, Aging and Cancer, Tohoku University (Sendai, Japan). K562 cells were cultured in RPMI 1640 medium supplemented with $10 \%$ FBS, $2.05 \mathrm{mM}$ glutamine, and $30 \mu \mathrm{g} / \mathrm{mL}$ kanamycin.

\section{Vector Construction}

pME-EGFP. The gene encoding enhanced green fluorescent protein (EGFP) was prepared from pEGFP (BD Biosciences Clontech, Palo Alto, CA, USA) by digestion with SmaI and $X b a I$. The fragment was inserted into the multiple cloning site of pBlueScript ${ }^{\circledR}$ SKII $\left(^{-}\right)$(Stratagene, La Jolla, CA, USA) between the $S m a \mathrm{I}$ and $\mathrm{XbaI}$ sites. The EcoRI and XbaI fragment encoding EGFP was then obtained from the vector by digestion with Eco$\mathrm{RI}$ and $X b a \mathrm{I}$ and inserted into the pME vector containing the promoter $\mathrm{SR} \alpha$ to give the plasmid pME-EGFP (about $6300 \mathrm{bp}$ ).

pEGFP-N1'. The gene encoding EGFP was prepared from pEGFP by digestion with HindIII and NotI. The HindIII-NotI fragment encoding EGFP was inserted into the HindIII and NotI sites of pDsRedII-N1 (BD Biosciences Clontech) to exchange for the gene coding EGFP instead of DsRedII, and the pEGFP-N1' expression vector (4731 bp) was obtained.

pDsRedII-N1. This expression vector was purchased from Invitrogen. The three expression vectors were separately purified using an EndoFree ${ }^{\circledR}$ Plasmid Mega Kit (QIAGEN GmbH, Hilden, Germany), according to the manufacturer's instructions.

Green fluorescent protein (GFP)- specific small interfering RNA (siRNA; GFP-22 siRNA) and fluorescein isothiocyanate (FITC)-labeled control siRNA [control (nonsilencing) siRNA, fluorescein] were purchased from QIAGEN K.K. (Tokyo, Japan).

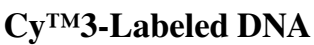

pEGFP-N1' was labeled with a Cy3 fluorescent dye using a Mirus Label IT $^{\circledR}$ Tracker $^{\mathrm{TM}}$ Cy3 kit (Mirus, Madison, WI, USA), according to the manufacturer's instructions.

\section{K562 Cells Stably Expressing EGFP}

The SacI-digested pME-EGFP expression vector, a linear plasmid, was introduced into K562 cells with LF2000. The transfectants were then cloned by limiting dilution. One of the cloned cells was used in the experiments.

\section{Immobilization and Transfection of K562 Cells on BAM-Modified Surfaces}

The surfaces of the glass slides were treated under aseptic conditions with $1 \%$ BSA in PBS at room temperature for $16 \mathrm{~h}$. After washing with Milli-Q ${ }^{\circledR}$ water (Millipore, Billerica, MA, USA), the surfaces were treated with $100 \mu \mathrm{M}$ BAM180 in PBS for $10 \mathrm{~min}$ at room temperature. The BAM-modified surfaces of the slides were washed with Milli-Q water and then air-dried at room temperature.

Cy3-labeled pEGFP-N1' $(1 \mu \mathrm{g})$ or unlabeled pEGFP-N1' $(1 \mu \mathrm{g})$, LF2000 ( $2 \mu \mathrm{L})$, RPMI 1640 medium $(8 \mu \mathrm{L})$, and Milli-Q water $(28 \mu \mathrm{L})$ were mixed and preincubated for $10 \mathrm{~min}$ at room temperature. Aliquots $(1 \mu \mathrm{L})$ of the mixture were manually spotted on the BAM-modified glass slides and airdried at room temperature for $30 \mathrm{~min}$ or incubated at room temperature for $30 \mathrm{~min}$ in high humidity. The diameter of the spots was about $1.5 \mathrm{~mm}$. The slides were then rinsed once with PBS. Nonadherent human erythroleukemia K562 cells suspended in serum-free RPMI 1640 medium $\left(5 \times 10^{5}\right.$ cells $\left./ \mathrm{cm}^{2}\right)$ were transferred onto the slides and incubated for approximately $10 \mathrm{~min}$ at room temperature. Subsequently, the 
cell-immobilized slides were gently washed with PBS to remove any nonimmobilized cells, and then the immobilized K562 cells on the slides were cultured in RPMI 1640 containing 10\% FBS $(25 \mathrm{~mL}$ per glass slide in a $10-\mathrm{cm}$ culture dish) for $42 \mathrm{~h}$ at $37^{\circ} \mathrm{C}$ in an atmosphere of $5 \% \mathrm{CO}_{2}$. The Cy3-labeled DNA and EGFP-expressing K562 cells on the BAM-modified slides were then observed using a conventional fluorescence microscope (IX81; Olympus K.K., Tokyo, Japan) and a chargecoupled device (CCD) digital camera (CoolSNAP ${ }^{\mathrm{TM}} \mathrm{HQ}$; Roper Industries, Duluth, GA, USA).

\section{Transfection of EGFP-Expressing K562 Cells with siRNA on BAM- Modified Glass Slides}

Approximately $1 \mu \mathrm{L}$ of $100 \mu \mathrm{M}$ BAM180 in PBS was spotted into about $2 \mathrm{~mm}$ diameter spots on BSA-precoated glass slides that had been prepared with BSA as described above, and the slides were incubated for $10 \mathrm{~min}$ at room temperature. The BAM-spotted surfaces of the slides were washed with PBS and air-dried at room temperature.

GFP-specific siRNA $(0.3 \mu \mathrm{g})$, LF2000 (1 $\mu \mathrm{L})$, RPMI 1640 medium $(2 \mathrm{~mL})$, and Milli-Q water $(6 \mu \mathrm{L})$ were mixed and preincubated for 30 min at room temperature. In the same way, a mixture including fluoresceinlabeled control siRNA instead of the GFP-specific siRNA was also prepared as a negative control. Aliquots $(1 \mu \mathrm{L})$ of the mixture solutions were separately spotted into about $1 \mathrm{~mm}$ diameter spots within the BAM-modified area of the slides and air-dried at room temperature for $30 \mathrm{~min}$. K562 cells stably expressing EGFP were suspended in serum-free RPMI 1640 medium $\left(3 \times 10^{5}\right.$ cells $\left./ \mathrm{cm}^{2}\right)$, and the cell suspension was plated onto the treated slides and incubated for approximately $10 \mathrm{~min}$ at room temperature. After gently washing with PBS, the immobilized EGFP-expressing K562 cells on the slides were cultured in RPMI 1640 containing 10\% FBS $(25 \mathrm{~mL}$ per glass slide in a $10-\mathrm{cm}$ culture dish) for $48 \mathrm{~h}$ at $37^{\circ} \mathrm{C}$ in an atmosphere of $5 \% \mathrm{CO}_{2}$. EGFP expression was then observed using a fluorescence microscope.

\section{Assessment of Cross-Contamination Between DNA Spots}

Two kinds of DNA/LF2000 complex solutions were prepared. pME-EGFP $(2 \mu \mathrm{g}), \mathrm{LF} 2000(1 \mu \mathrm{L}), \mathrm{RPMI} 1640 \mathrm{me}-$ dium $(2 \mu \mathrm{L})$, and Milli-Q water $(5 \mu \mathrm{L})$ were mixed and preincubated for 30 $\mathrm{min}$ at room temperature (pME-EGFP solution). Likewise, a pDsRedII-N1 solution in which $1 \mu \mathrm{g} / \mu \mathrm{L}$ pDsRedII-N1 $(2 \mu \mathrm{L})$ was used instead of the pMEEGFP was also prepared.

Each gene solution (about $0.1 \mu \mathrm{L}$ ) was separately spotted on a BAM180modified coverslip. The spots were about $1 \mathrm{~mm}$ in diameter with an edgeto-edge distance of about $0.5 \mathrm{~mm}$. A K562 cell suspension in serum-free RPMI 1640 medium $\left(6 \times 10^{5}\right.$ cells $\left./ \mathrm{cm}^{2}\right)$ was transferred onto the BAM-modified coverslip where the plasmid DNAs had been spotted and dried and incubated for approximately $10 \mathrm{~min}$ at room temperature. Subsequently, the nonimmobilized cells were removed by gentle washing with PBS, and the K562 cells immobilized on the coverslip were cultured in RPMI 1640 containing 10\% FBS for 48 $\mathrm{h}$ at $37^{\circ} \mathrm{C}$ in an atmosphere of $5 \% \mathrm{CO}_{2}$. The expression of each gene in the immobilized cells was observed using a fluorescence confocal laser-scanning microscope (TCS-NT ${ }^{\circledR}$; Leica Microsystems K.K., Tokyo, Japan)

\section{Fabrication of a Cell Microarray Using an Ink-Jet Printer}

A BAM180-modified glass slide and the two kinds of DNA/LF2000 complex solutions (pME-EGFP and pDsRedIIN1) were prepared as described above. Spots of each gene solution $(20 \mathrm{~nL})$ were microprinted onto the BAM180modified glass slide at room temperature using an ink-jet printer (Cartesian Technologies, Irvine, MI, USA), according to one of the following two patterns. Pattern 1 was composed of 88 spots of only the pDsRedII-N1 complex solution, while pattern 2 was composed of a checkered pattern formed from the pME-EGFP and pDsRedII-N1 solutions. All the spots were approximately $500 \mu \mathrm{m}$ in diameter, and the spot centers were $1 \mathrm{~mm}$ apart. These gene-printed slides were stored at $4^{\circ} \mathrm{C}$ in the presence of a desiccating agent until use.

K562 cells suspended in serumfree RPMI 1640 medium $\left(6 \times 10^{5}\right.$ cells $/ \mathrm{cm}^{2}$ ) were transferred onto the gene-printed slides and incubated for approximately $30 \mathrm{~min}$ at room temperature. After gentle washing and culturing for $48 \mathrm{~h}$ in RPMI 1640 containing $10 \%$ FBS and $90 \mu \mathrm{g} / \mathrm{mL}$ kanamycin, the immobilized and gene-expressing cells were photographed using a fluorescence microscope and shown as tiled images.

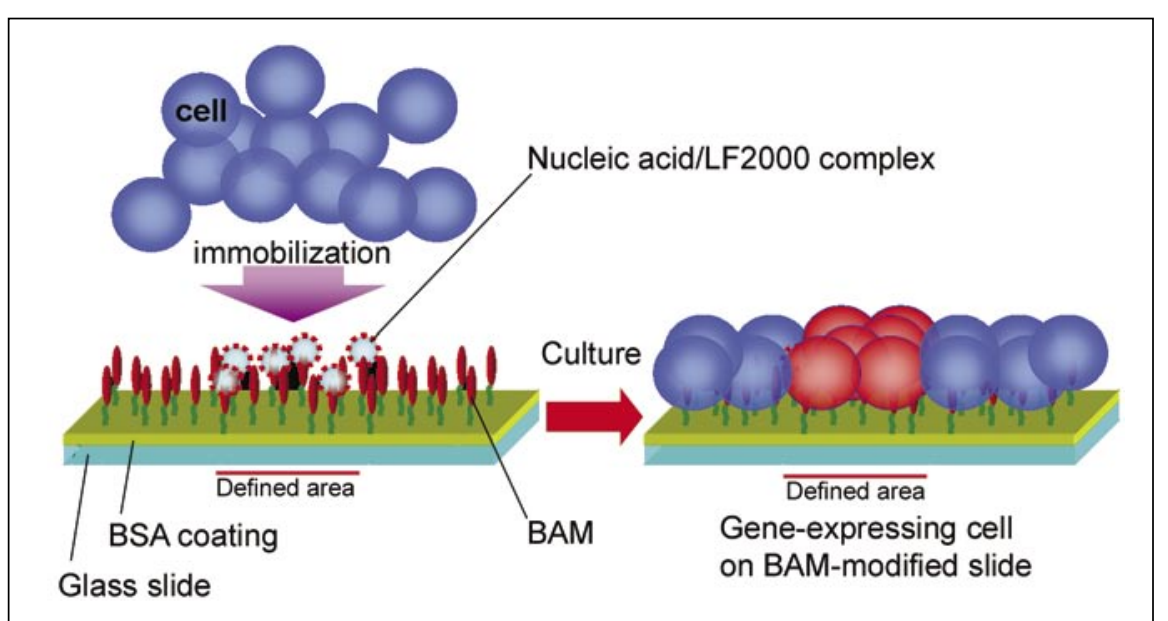

Figure 1. Transfection microarray of nonadherent cells. Biocompatible anchor for membrane (BAM) 180 couples with the amino groups of bovine serum albumin (BSA) adsorbed on a glass slide. Liposomes of Lipofectamine 2000 binding DNA are noncovalently immobilized to the BAM-modified surface by the interaction between the liposome membrane and the oleyl group of BAM. Likewise, nonadherent cells are also immobilized on the BAM-modified surface binding Lipofectamine 2000. The immobilized cells within the area defined by the gene spot can be transfected with the proximate DNA/ Lipofectamine 2000 complexes and subsequently express the gene product. BSA, bovine serum albumin; LF2000, Lipofectamine 2000. 


\section{RESULTS}

\section{Transfection of Cells with Plasmid DNA on BAM-Modified Glass slides}

In an earlier report, we showed that liposomes as well as cells attach to the BAM-modified glass surface (8). From this result, it was expected that cells and liposomes could be simultaneously immobilized at the same place on the BAM surface. To transfect cells with the genes in a small, defined area on the surface of a BAM-modified glass slide, we used a cationic liposome transfection reagent, LF2000, because we expected that DNA electrostatically bound to liposomes would be immobilized at the defined area on the BAM surface. Figure 1 shows the transfection scheme of the immobilized cells with plasmid DNA/liposome complexes on the BAM surface.

To test whether it was possible to attach DNA and introduce it into cells immobilized at the same place on the BAM surface, we spotted pEGFP-N1' plasmid DNA/LF2000 complexes on a BAM-modified glass slide and then added K562 cells and allowed them to become immobilized on the slide. In this experiment, we prepared two spots of DNA/LF2000 complexes with different treatments after the spotting. In one, the spotted solution was air-dried (dried spot), while in the other, drying was avoided by using a high humidity atmosphere (wet spot). After K562 cells had been immobilized and cultured for $48 \mathrm{~h}$ on the BAM surface, including each spot area, EGFP expression in the cells was observed by fluorescence microscopy. We found that K562 cells immobilized on the dried spot expressed EGFP within the area defined by the spotting of the DNA/LF2000 complexes (Figure 2, indicated by a red circle), and the proportion of cells expressing EGFP was about 40\% (Figure 2A). By contrast, in the K562 cells immobilized on the wet spot, the proportion of EGFP expression was low, and EGFP-expressing cells were also located outside of the DNA/LF2000 complex spotting area (Figure 2D). These results (Figure 2D) indicate that the DNA/LF2000 complex diffused from the spotted area. This finding differs from the case of the DNA/LF2000 complex solution dried on the BAM surface, where it appears that most of the liposomes in the DNA/ LF2000 solution were stably immobilized on the dry-spotted area. To confirm and directly compare the amounts of DNA immobilized at the spotted areas of the two spotting protocols, a DNA/LF2000 mixture including Cy3-
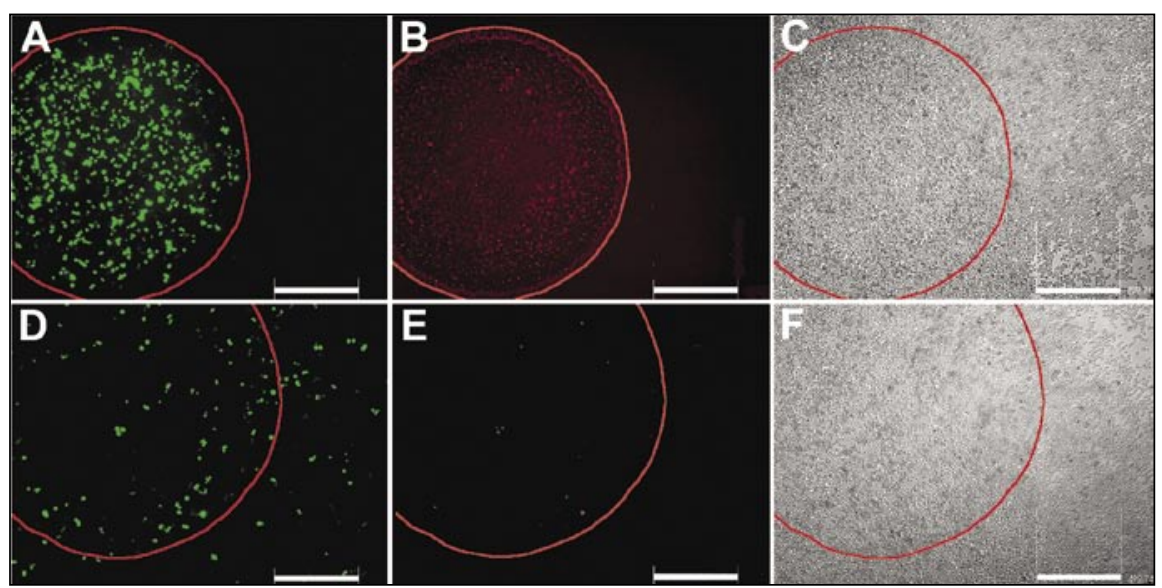

Figure 2. Gene expression in human erythroleukemia K562 cells immobilized on a biocompatible anchor for membrane (BAM)-modified glass slide. A solution of pEGFP-N1' plasmid and Lipofectamine 2000 complexes was spotted on the BAM180-modified glass slide within the areas indicated by the red circles. (A-C) After drying the solution, K562 cells were immobilized on the slide and cultured for $48 \mathrm{~h}$. (D-F) After incubating the solution on the BAM-modified slide for $30 \mathrm{~min}$ without drying using a high humidity atmosphere, the cells were immobilized and cultured for $48 \mathrm{~h}$. (A and D) Immobilized K562 cells expressing green fluorescent protein (GFP) after $48 \mathrm{~h}$ (fluorescence images). (B and E) Cy3-labeled pEGFP-N1 deposited on the slide, which was incubated for $48 \mathrm{~h}$ in RPMI 1640 containing $10 \%$ fetal bovine serum (FBS) (fluorescence images). (C and F) K562 cells immobilized and cultured on the slide for $48 \mathrm{~h}$ (bright-field images). All scale bars indicate $500 \mu \mathrm{m}$. 
labeled DNA was used. The amount of DNA in the dried spot was higher than that in the wet spot (Figure 2, B and E), although the amounts of both DNAs decreased with increasing incubation time in the culture medium. Thus, the EGFP expression levels depended on the amount of DNA immobilized on the BAM-modified slide. In addition to the advantages of the dried spot protocol for spot integrity, the dried gene-arrayed slides appear to be favorable for preservation. We have some preliminary data indicating that it is possible to use DNA/LF2000-spotted slides that have been preserved for 253 days at $4^{\circ} \mathrm{C}$ in the presence of a desiccating agent (data not shown).

\section{Transfection of Cells with siRNA on the BAM-Modified Surface}

Within the context of cell-based microarrays, studies using RNA interfer- ence (RNAi) are an attractive approach for analyzing gene functions in vivo by observing knockdown phenotypes in the cells (3-4). We investigated whether we could achieve silencing of gene expression using our method to transfect immobilized cells with siRNA. We first spotted a complex consisting of siRNA corresponding to the EGFP gene (siRNA/EGFP) and LF2000 and then immobilized K562 cells stably expressing EGFP (K562/EGFP cells) on BAM-modified slides. As a result, silencing of the EGFP expression in K562/EGFP cells was observed within the spot containing the siRNA/EGFP and LF2000 complexes (Figure 3A). The EGFP expression in K562/EGFP cells outside of the siRNA spot was not affected. Furthermore, the EGFP expression in K562/EGFP cells cultured on a spot of control siRNA was unaffected and was the same as that of the cells outside the spot (Figure 3B).

\section{Assessment of Cross-Contamination Between Adjacent DNA Spots}

As the results in Figure 2A had indicated, LF2000/EGFP gene complexes spotted on BAM180-modified glass slides could be incorporated into nonadherent K562 cells immobilized on the slide, leading to spatially defined cells with EGFP expression. Next, we analyzed the potential for cross-contamination of transfected K562 cells with different genes that were deposited close to each other (500 $\mu \mathrm{m}$ apart). We observed that immobilized K562 cells were uniquely transfected with either pME-EGFP or pDsRedII-N1 at the corresponding regions defined by spots of each gene on a BAM-modified coverslip without cross-contamination (Figure 4A). The proportions of gene-expressing cells on the gene spots were about $30 \%-50 \%$.

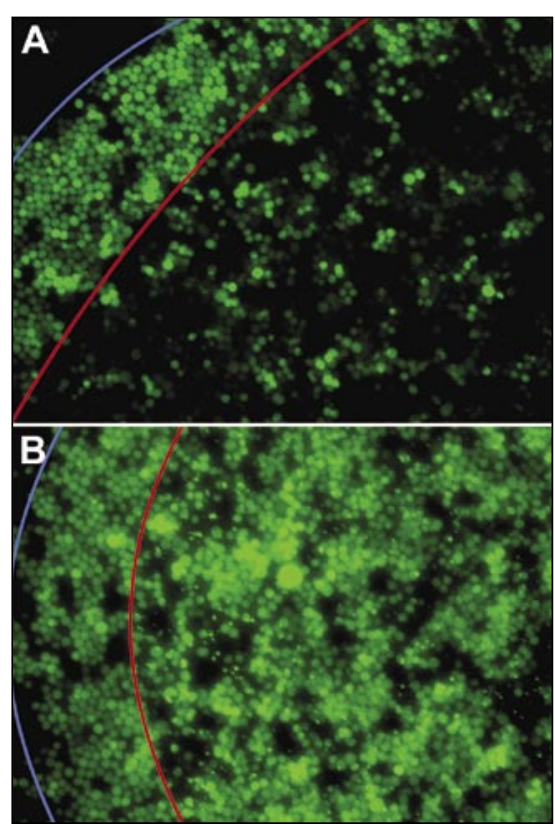

Figure 3. Fluorescence photomicrographs of EGFP gene silencing in $\mathrm{K562}$ cells immobilized on biocompatible anchor for membrane (BAM)180-modified surfaces. Inside the blue line is the BAM180-modified area on the coverslip [the whole area was precoated with bovine serum albumin (BSA)]. The area inside the red line was treated with small interfering RNA (siRNA). (A) GFP-specific siRNA and Lipofectamine 2000 (LF2000) complex solution. (B) Control siRNA and LF2000 complex solution. K562 cells stably expressing EGFP were immobilized and cultured for $48 \mathrm{~h}$ on the treated surfaces. EGFP, enhanced green fluorescent protein.

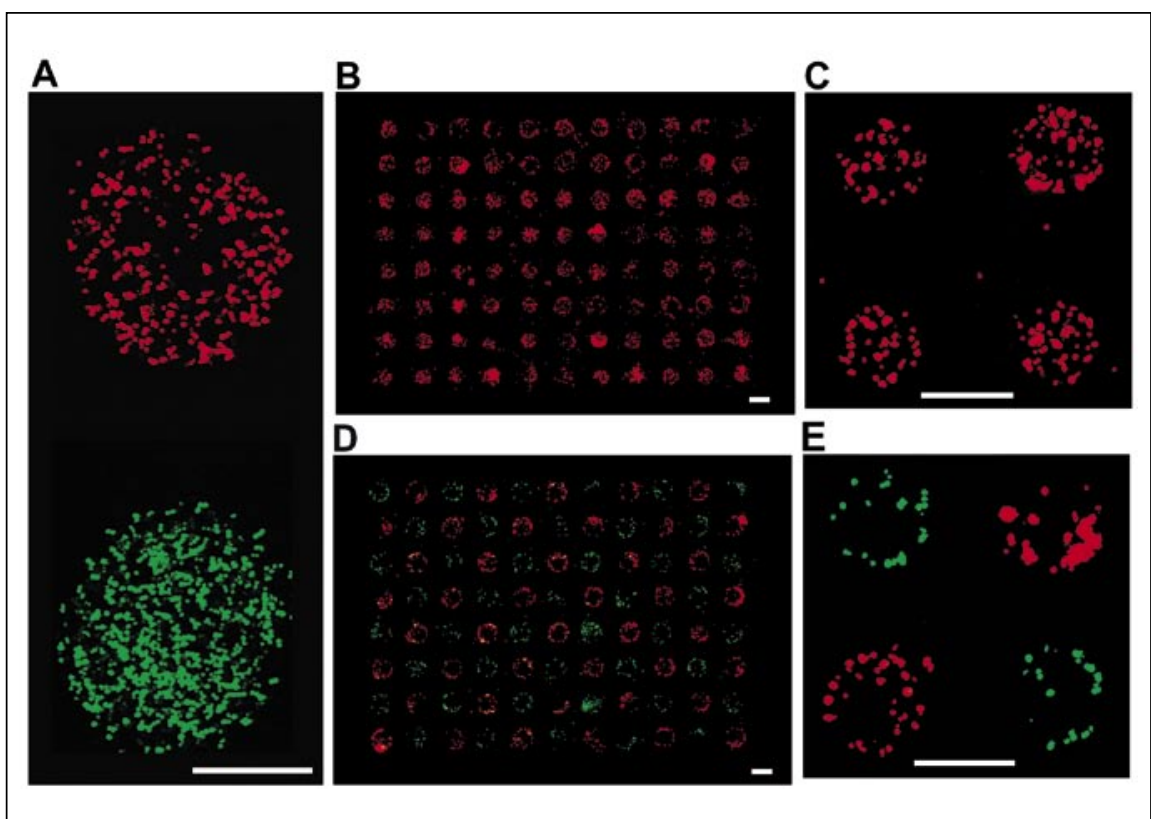

Figure 4. High-density transfection microarray of nonadherent K562 cells. (A) Separated expression of two different genes (pME-EGFP and pDsRedII-N1) within separately defined areas on the same surface. DNA/Lipofectamine 2000 complex solutions (about $0.1 \mu \mathrm{L}$ ) were spotted by manual pipetting. These spots were about $1 \mathrm{~mm}$ in diameter, and the edge-to-edge distance was about $500 \mu \mathrm{m}$. K562 cells were immobilized and cultured for $48 \mathrm{~h}$, and the fluorescence of enhanced green fluorescent protein (EGFP) expressions was observed using a fluorescence confocal laser-scanning microscope. (B-E) Transfection microarrays of nonadherent K562 cells on biocompatible anchor for membrane (BAM)180modified glass slides. DNA and Lipofectamine 2000 complex solutions were microprinted using an ink-jet printer. Each spot $(20 \mathrm{~nL})$ was $500 \mu \mathrm{m}$ in diameter, and the spot centers were $1 \mathrm{~mm}$ apart. (B) pDsRedII-N1 array. (C) Partial high-magnification image of panel B. (D) pDsRedII-N1 and pME-EGFP (checkered pattern) array. K562 cells were immobilized and cultured for $48 \mathrm{~h}$. (E) Partial high-magnification image of panel D. All scale bars indicate $500 \mu \mathrm{m}$. 


\section{High-Density Transfection Micro- array of Nonadherent K562 Cells}

Based on the above studies, we attempted to fabricate a high-density transfection microarray for nonadherent cells. First, the pDsRedII-N1/ LF2000 complex solution was microprinted on a BAM180-modified glass slide using an ink-jet printer $(20 \mathrm{~nL} /$ spot). The array was constructed of $8 \times 11$ spots of $500 \mu \mathrm{m}$ in diameter, and the centers of the spots were 1 $\mathrm{mm}$ apart. This format theoretically allows for an array of 1875 spots on a standard glass slide $(76 \times 26 \mathrm{~mm})$, and it is possible to array more spots depending on the performance of the arrayer device. The addition of a cell suspension to the surface of the gene-arrayed slide resulted in the immediate immobilization of the cells. After culturing for $48 \mathrm{~h}$, the DsRedII expression was photographed using a fluorescence microscope, and the pho- tos were arranged as tiled images (Figure 4, B and C). About 100-150 cells expressed the DsRedII fluorescence protein in a spot, and the proportions of expressing cells were no more than $10 \%-15 \%$.

Next, two different DNA/LF2000 complex solutions, pME-EGFP and pDsRedII-N1, were printed in a checkered pattern using the ink-jet printer. We observed that the proportion of gene expression was approximately $10 \%$, and that almost all the transfected cells were localized within the area defined by the gene spots (Figure 4, D and E). Further, all cell clusters on the gene spots were expressing either the EGFP or DsRedII.

\section{DISCUSSION}

Our studies indicate that it is possible to introduce cDNA-expressing plasmid DNA into cells immobilized on the
BAM surface. We accomplished this by immobilizing nonadherent cells on a BAM-modified surface that had been spotted with a complex of DNA and the cationic liposome, LF2000.

If the DNA/LF2000 complex was directly deposited on the glass slide surface, suspended cells were temporarily adsorbed to the surface of the deposited complex by the positive electrostatic charge of LF2000. However, the cells adsorbed on the complex-deposited surface dissociated when the complex was dissolved in culture medium (data not shown). On the other hand, cells immobilized on the BAM-modified surface spotted with the DNA/LF2000 complex did not dissociate during a culture period of about $72 \mathrm{~h}$.

For the immobilization of the DNA/ LF2000 complex on the BAM surface, the complexes had to be dried. When complex solutions were not sufficiently dried, nonbinding liposomes in the spotted solution spread out of the spotted 
areas during washing. Fortunately, the dried DNA/LF2000 complexes were suitable for the transfection of immobilized cells. Furthermore, dried complexes on a BAM surface stored for 253 days under conditions of low humidity at $4^{\circ} \mathrm{C}$ still showed high efficiency for transfection of immobilized cells.

Gene expression levels were not high in the experiments performed with the high-density transfection microarray. We speculate that there are two reasons why the gene expression levels decreased. First, the DNA/LF2000 complex solution printed by the inkjet printer was heterogeneously dried without humidity control, and therefore the DNA/LF2000 complexes were not homogeneously distributed in the spots. That is, because the volume of printing solution was very small, the solutions dried immediately. This was especially true near the center of spot, where the solution would be dried before the DNA/LF2000 complex was sufficiently immobilized. As a result, the DNA/LF2000 complex would become condensed at the edge of the spot, and the distribution of the DNA/LF2000 complex would become heterogeneous in the spot. By contrast, the spots that were manually spotted did not develop a heterogeneous distribution of DNA/ LF2000 because the volume of the solution was more than that desposited by the ink-jet printer.

Second, we could not aseptically prepare the gene microarray slides, and thus a high concentration of antibiotic (90 $\mu \mathrm{g} / \mathrm{mL}$ kanamycin) was required to maintain sterility. The high concentration of antibiotic might have reduced the level of gene expression. However, these problems should be resolved by operating under circumstances where humidity, temperature, and sterility are well controlled.

Further optimization may also be necessary to adapt this reverse transfection method to other suspension cells. Preliminary studies with Jurkat $\mathrm{T}$ cells showed a low $(<1 \%)$ proportion of gene-expressing cells. In addition, many nonadherent cells, such as CTLL-2 lymphocytes and Ba/F3 cells, are not efficiently transfected with LF2000 and thus would not be expected to result in high-level gene expression using this reverse transfection method. Further studies will be needed to develop effective reverse transfection strategies for these cell types. It should be noted that the method is not limited to adherent cells, as HEK293 cells were transfected with about $40 \%$ efficiency using a slightly modified method (data not shown).

In the high-density transfection microarray experiment, we used an inkjet printer as our noncontact arrayer. However, it might be possible to use a contact pin arrayer because our manual spotting experiments showed that we could spot DNA/LF2000 complex solutions by tapping pipet tips against the BAM surface; no problems such as detachment of the BAM surface were observed.

This new reagent, BAM, allowed for the creation of a cell-based microarray for nonadherent cells, and thus the current limitation of cell-based microarray methods to adherent cells has been eliminated. This technique will greatly expand the scope of cell microarray technology.

\section{ACKNOWLEDGMENTS}

We thank T. Yasukouchi, C. Ito, S. Ohhashi, K. Kubo, and other members of the NOF Corporation for the synthesis of the BAMs and PEG-NHS. We thank Dr. K. Yokoyama and T. Fukumori (Research Center of Advanced Bionics, National Institute of Advanced Industrial Science and Technology, Tsukuba, Japan) for microprinting of the genes using an ink-jet printer.

\section{COMPETING INTERESTS STATEMENT}

The authors declare that they have no competing interests.

\section{REFERENCES}

1.Bailey, S.N., R.Z. Wu, and D.M. Sabatini. 2002. Applications of transfected cell microarrays in high-throughput drug discovery. Drug Discov. Today 7:S113-S118.

2.Howbrook, D.N., A.M. van der Valk, M.C. O'Shaughnessy, D.K. Sarker, S.C. Baker, and A.W. Lloyd. 2003. Developments in microarray technologies. Drug Discov. Today 8:642-651.
3.Kumar, R., D.S. Conklin, and V. Mittal. 2003. High-throughput selection of effective RNAi probes for gene silencing. Genome Res. 13:2333-2340.

4.Mousses, S., N.J. Caplen, R. Cornelison, D. Weaver, M. Basik, S. Hautaniemi, A.G. Elkahloun, R.A. Lotufo, et al. 2003. RNAi microarray analysis in cultured mammalian cells. Genome Res. 13:2341-2347.

5.Mousses, S., A. Kallioniemi, P. Kauraniemi, A. Elkahloun, and O.P. Kallioniemi. 2002. Clinical and functional target validation using tissue and cell microarrays. Curr. Opin. Chem. Biol. 6:97-101.

6.Wu, R.Z., S.N. Bailey, and D.M. Sabatini. 2002. Cell-biological applications of transfected-cell microarrays. Trends Cell Biol 12:485-488.

7.Ziauddin, J. and D.M. Sabatini. 2001. Microarrays of cells expressing defined cDNAs. Nature 411:107-110.

8.Kato, K., K. Umezawa, D.P. Funeriu, M. Miyake, J. Miyake, and T. Nagamune. 2003. Immobilized culture of nonadherent cells on an oleyl poly(ethylene glycol) ether-modified surface. BioTechniques 35:1014-1021.

Received 23 February 2004; accepted 19 May 2004.

Address correspondence to:

Teruyuki Nagamune

Department of Chemistry and Biotechnology

School of Engineering, The University of Tokyo

7-3-1, Hongo, Bunkyo-ku

Tokyo, 113-8656, Japan

e-mail: Nagamune@bio.t.u-tokyo.ac.jp 